FERMILAB-FN-0822-CD-T

May 20, 2008

(rev. 6/8/08)

\title{
Direct Observation Limits on Antimatter Gravitation
}

\author{
Mark Fischler*, Joe Lykken, and Tom Roberts ${ }^{\dagger}$
}

June 8,2008

\begin{abstract}
The proposed Antihydrogen Gravity experiment at Fermilab (P981) will directly measure the gravitational attraction $\bar{g}$ between antihydrogen and the Earth, with an accuracy of $1 \%$ or better. The following key question has been asked by the PAC:

Is a possible $1 \%$ difference between $\bar{g}$ and $g$ already ruled out by other evidence?

This memo presents the key points of existing evidence, to answer whether such a difference is ruled out (a) on the basis of direct observational evidence; and/or (b) on the basis of indirect evidence, combined with reasoning based on strongly held theoretical assumptions. The bottom line is that there are no direct observations or measurements of gravitational asymmetry which address the antimatter sector. There is evidence which by indirect reasoning can be taken to rule out such a difference, but the analysis needed to draw that conclusion rests on models and assumptions which are in question for other reasons and are thus worth testing. There is no compelling evidence or theoretical reason to rule out such a difference at the $1 \%$ level.
\end{abstract}

*Fermi National Accelerator Laboratory

${ }^{\dagger}$ Muons, Inc. and Illinois Institute of Technology 


\section{Contents}

1 The Nature of Evidence About Gravitational Asymmetry 3

2 Limits Set By Force or Acceleration Observations 5

$\begin{array}{llr}3 & \text { Limits Based on Time-Scaling Effects } & 7\end{array}$

4 Inferences From Astronomical (Anti)Neutrino Observations 10

5 Theoretical Obstacles to Gravitational Asymmetry 10

6 Conclusions 12 


\section{The Nature of Evidence About Gravita- tional Asymmetry}

P981 is a proposed Fermilab experiment [1] to measure, using atomic beam interferometry, the gravitational acceleration of antihydrogen. The experimenters are confident that $1 \%$ accuracy or better would be achieved. During an initial presentation to the PAC, the following question was posed:

Is a possible $1 \%$ difference between $\bar{g}$ and $g$ already ruled out by other evidence?

We take this as asking whether a difference of that magnitude is ruled out either by direct observation, or by experimental results that lead to incontrovertible (though indirect) evidence. If experimental results exist that cannot be reconciled with this level of gravitational asymmetry, assuming any sensible theory, then one could say the asymmetry is ruled out. We also consider whether any known theoretical consideration (based, for instance, on some gedanken experiment) irrefutably rules out such an effect.

Of course, one might simply assert that the only "sensible" theory at low energies and macroscopic distances is standard General Relativity with the Weak Equivalence Principle holding exactly, and further assert that given this framework, such an asymmetry is impossible. There are three major weaknesses of this "proof by assertion":

- There is evidence (from the accelerating expansion of the universe ) that calls into question either the composition of the universe or the correct theory to describe the interactions driving that expansion. It is not out of the question that a revised theory correctly describing the expansion could include interactions that distinguish antimatter from matter.

- The simplest theoretical model that predicts a non-null result for the antihydrogen experiment postulates new vector and scalar mediated forces that couple to some combination of baryon and lepton number (or any other quantity that distinguishes matter from antimatter). If the vector and scalar forces are of equal magnitude, their effects will to some high accuracy cancel for ordinary matter. Nieto and Goldman [2] (section 8) point out that in this context, "the more precisely anomalous gravitational effects are ruled out in earth-based matter-matter experiments, the more unrestricted is the possibility that there can be a significant anomalous gravitational acceleration of antimatter." These "fifth forces" do not invalidate General Relativity any more than do the gauge forces of the Standard Model.

- One must always be careful about hidden assumptions. One obviously relevant hidden assumption is CPT symmetry. While this can be proven rigorously for asymptotically flat-space relativistic local field theories, string theory calls into question both locality and the fundamental existence of an S-matrix.

We will discuss in section 5 whether there is general agreement among the gravitational theory community that antimatter gravitational asymmetry can or 
cannot be accommodated by a consistent theory. First, however, we will examine consequences of existing down-to-earth measurements and observations directly or indirectly bearing on this question.

\subsection{Meaning of "Direct Observation"}

What constitutes a "direct observation" of the value of some physical quantity $(\bar{g} / g$ in this case)? We want to avoid general philosophic discussions, so we will define a "direct observation" of a value as evidence from which the value can be deduced without depending on any assumptions which a reasonable and expert scientist might call into question. This implies that the analysis can be done in a straightforward and essentially model-independent manner.

In the case at hand, we are asked to address whether $\bar{g} / g \neq 1$ is ruled out at the $1 \%$ level by existing evidence.

\subsection{Categories of (Experimental) Evidence Against Gravitational Asymmetry}

Although there are multiple experiments measuring asymmetry between one sort of particle and another, in relation to gravity, they all fall into three general classes. First, there are experiments that drop, throw, or (gravitationally or otherwise) deflect different types of matter and measure the resulting paths or forces [3] [4] [5] [6] [7]. These can be considered to be "direct" measurements, since they address the quantity (force or acceleration) in which we are specifically interested, and depend on no framework (other than that needed to arrive at the force or acceleration results themselves) to interpret their data. We can think of this as "Newtonian" evidence, since such results can bear on the issue without any framework beyond $F=m a$. Direct observations are discussed in section 2 .

Second, there are experiments (or deductions made incidental to other experiments) for which analysis of certain negative results allows one to "rule out" gravitational asymmetry at some level, by applying some properties of gravity [11] [12] [13] [14]. The crucial property in these experiments involves gravity rescaling a particle's observed time relative to lab time. These can be considered to be "indirect" measurements, since one must use at least some generic property of the underlying gravitational theory to derive the asymmetry limits from their data. We can think of this as "Einsteinian" evidence, since such results impact the issue of gravitational asymmetry via concepts that are not present in non-relativistic physics. These deductions are discussed in section 3 .

A third group of arguments is based on astrophysical evidence about neutrinos and antineutrinos: observation of equal time-of-flight for neutrinos and antineutrinos originating in supernova SN1987A [16], and inferences from solar [17] or terrestrial [18] neutrino oscillation observations. We will see in section 4 that these arguments are uniformly weaker evidence against the possibility of antimatter gravitational asymmetry than the "Einsteinian" evidence. 


\subsection{What Fraction of the Antiproton Mass Is Relevant?}

An observation has been made [19] that even if $\bar{g} / g=-1$, the antiproton would not "fall up" with acceleration $g$. This is because a large fraction of the inertial mass of a proton or antiproton comes from its binding energy (the gluon field). While it is unclear whether to use the low momentum current-quark masses or some other quantity (perhaps a chiral mass) as the component which distinguishes between matter and antimatter, if we say that the current-quark mass is critical, then the mass in question is only about $1 \%$ of the overall antiproton mass. Taking that viewpoint, the $1 \%$ level represents a maximum plausible effect.

We cannot refute that viewpoint, but it does depend on the Standard Model, testing of which has been the mission of Labs world-wide for several decades. Validation of predictions of the Standard Model in circumstances involving gravity is certainly fair game for investigation.

We note that the same $1 \%$ argument (that most of the antiproton mass consists of "ordinary" binding energy) applies to the analysis of all the experimental evidence we will be looking at below, and weakens every statement the experiments make about limits on $\bar{g} / g$. However, the most precise experimental evidence of each type sets a limit (if accepted as conclusively bearing on $\bar{g}-g$ ) which is much smaller than $1 \%$.

The bottom line about this "quark fraction" reasoning is that it is moot to the issue of whether a $1 \%$ effect can possibly be present in antihydrogen measurements: If either class of experimental results is accepted as placing irrefutable or direct limits on $\bar{g}$, then the proposed experiment cannot detect an effect, even ignoring the "quark fraction" reasoning. And if none of that evidence presents a conclusive measurement, then there is potential for an effect as large or larger than the $1 \%$ level.

\section{Limits Set By Force or Acceleration Obser- vations}

A $\bar{p}$ trajectory experiment sensitive to gravity would provide a direct measurement of the value of $\bar{g}$. This sort of experiment was proposed at Fermilab [20] and suggested as part of an experiment at LEAR [21], but measurement of the trajectory of (charged) antiprotons has never been done with sufficient sensitivity to observe gravitational acceleration. There have been neutron free-fall experiments [5] [7], which verify that neutrons have the same value of $g$ as ordinary neutron-proton matter, to an accuracy of $0.2 \%$. Again, such an experiment with antineutrons would constitute a direct observation of $\bar{g} / g$, but that hasn't been done either, and is not likely to be feasible because antineutrons can't easily be cooled - any cooling mechanism involving matter interaction turns into an annihilation mechanism instead. The most sensitive neutron experiment that has been performed involved bouncing neutrons on a matter surface, and thus is inherently impossible to replicate with antineutrons. 
Eötvös-style experiments [6], measuring the gravitational attraction between a heavy mass and test masses of various element compositions, directly measure the difference in coupling to elementary particle mass and to binding energy. For example, the binding energy fraction for beryllium is half a percent less than that for copper, and the experimental limit on $|\Delta a / a|$ for beryllium versus copper is $2.5 \cdot 10^{-12}[4][3]$. This puts a limit on the difference of gravitational attraction for ordinary proton-neutron mass to nuclear binding energy of about $5 \cdot 10^{-10}$, and this has to be considered as direct observation, based on a force measurement.

At any rate, neither the Eötvös-style experiments nor the neutron free-fall experiments make any statement about antimatter, unless one stretches to say that different elements have different virtual antimatter proportions. The argument would go as follows: Suppose some fraction of the mass of the proton is attributable to masses of the (virtual) anti-quarks in the "sea." Then there will be analogous antimatter fractions in various nuclei. These antimatter fractions would be expected to have about the same value as that for the proton, but are unlikely to match precisely. Therefore, the elements tested will likely have antimatter fractions which differ by some (second-order small) amount. If this difference is as large as one part in a million, then the Eötvös-style experiments set a limit on antimatter gravitation asymmetry at about $5 \cdot 10^{-3}$.

This argument is greatly weakened by the cosmological constant problem. If gravity couples to virtual particles and antiparticles inside nucleons, it presumably also couples to virtual particles and antiparticles that constitute vacuum energy. This leads to the famous estimate of the cosmological constant that disagrees with observation by (at least) 120 orders of magnitude. Obviously there is something fundamentally wrong with our understanding of how to relate gravity to virtual effects.

Furthermore, even if the naive picture of gravity coupling to virtual antiparticles were correct, there is no strong justification for saying that the antimatter content fraction differs between one element and another, and certainly no estimate has been derived of the size of this putative effect.

So, although direct acceleration observations do fix gravitational indifference to the nature of mass (among ordinary matter and binding energy) at much better than the $1 \%$ level, they do not constitute direct observations of this property applied to antimatter.

\subsection{Combining Eötvös-style measurements with infer- ences from other observations}

A stronger line of reasoning combines the exquisite precision of Eötvös-style ordinary-matter measurements with results from other gravitation experiments and assumptions about the forms of possible equivalence-principle-violating interactions, to infer limits on the antimatter sector from those results. Detailed by the Eöt-Wash collaboration in section $\mathrm{F}$ of [6], and expounded more succintly in [9] and [8], the argument is: 
1. Assume deviation from the weak equivalence principle takes the form of vector and scalar gravity-like forces, where the vector force may affect antimatter differently from matter.

2. The Eöt-Wash results, by comparing elements with different proton-neutron fractions, force very precise cancellation between these gravi-scalar and gravivector forces in the ordinary matter sector.

3. Dimensional considerations restrict the nature of the vector and scalar forces to forms containing a limited set of parameters. The cancellation in the Eötvös-style experiments strongly constrains the relative values of some of these parameters.

4. Incorporation of the observational limit on difference between the proton and anti-proton $|q / m|$, and of laser geodynamics data that place a limit on deviation from $1 / r^{2}$ behavior, then places limits in other realms. These overconstrain the parameters unless the "cancelling" scalar and vector forces are very small.

This reasoning leads to a limit on the value of antihydrogen gravitational asymmetry, at the $10^{-5}$ level.

However, Goldman et al. [10] points out a weakness in the third point of the above argument: The restrictions on possible forms for the scalar and vector interactions stem from dimensional constraints which are applicable to renormalizable theories. These do not generally apply to gravitation theories. Loosening these restrictions leaves open the possibility of near-precise cancellation for ordinary matter, enforced by some approximate symmetry. And to the extent that such cancellations are possible, the Eötvös-style experiments do not constitute direct observations of gravitational indifference to the nature of mass where antimatter is concerned.

\section{Limits Based on Time-Scaling Effects}

There are two experiment-based arguments that put sensitive limits on matterantimatter gravitational asymmetry: the (tiny value of the) $K^{0}-\bar{K}^{0}$ oscillation rate [11] [12] [15], and the equality of cyclotron frequencies for the proton and the antiproton [13] [14].

We discuss these together because they both hinge on the general-relativistic effect of gravitational redshift. By this, we mean the effect that to an observer at the top of some gravitational potential, clocks on objects at a lower potential appear to tick more slowly. A consequence of the Weak Equivalence Principle (objects travel along geodesics), it also has been verified by direct observation of redshifts (e.g., downward traveling photons and shifts of solar spectral lines; see table 2.3 of reference [4]) matching the predicted value.

The cyclotron frequency evidence is particularly on-point for antihydrogen. This is discussed by Hughes and Holzscheiter [13]. The idea is that the cyclotron frequency for a proton depends on the proton's electromagnetic properties (mostly 
charge, but at higher orders perhaps magnetic moment) and mass. The cyclotron frequency is a rest-frame quantity. Since the mass and absolute charge of a $\bar{p}$ are identical to those of a $p$, the inherent cyclotron frequency will be identical. However, the quantity that external measurers will observe as the cyclotron frequency might not be identical if the time unit for a $\bar{p}$ is different from that for a $p$ !

Now let's place the $p$ (or $\bar{p}$ ) into a gravitational field as follows: The metric is asymptotically flat, but contains a concentration of mass centered at some distance $R$ from the proton. Relative to an observer near the concentration of mass, the local time of the proton is sped up by a factor of $1+G M / R c^{2}$. That is, there is a time acceleration effect proportional to the "absolute gravitational potential" felt by the proton.

Next, assume the $\bar{p}$ interacts differently with that gravitational field. Its time acceleration factor will instead be $1+(\bar{g} / g)\left(G M / R c^{2}\right)$. Thus its cyclotron frequency, as observed by "we the accelerator people" (who being made of ordinary matter will share the acceleration of the proton), will be different by an amount proportional to $(1-\bar{g} / g)\left(G M / R c^{2}\right)$. Such a difference would (in the experiments described by Gabrielse [14]) manifest as a difference in charge-to-mass ratio $|q / m|$ for the proton and antiproton.

What should we use for $R$ and $M$ if such an argument is true? Well, to a Good approximation (pun intended [11]), the local galactic super-cluster sits isolated in an asymptotically flat space, and produces a fairly large absolute gravitational potential $\left(\phi_{g}=G M / r c^{2} \approx 3 \times 10^{-5}\right)$ [12]. Since we know the proton and antiproton have the same cyclotron frequency to a part in $10^{10}$ [14], this sets an upper bound on $|1-\bar{g} / g|$ of about $5 \times 10^{-4}$.

Similarly, the observation of near-perfect non-regeneration of $K_{S}$ from the $K_{L}$ state (the small regeneration is observation of CP violation) would be utterly destroyed by differences in the local time steps between the $K^{0}$ and the $\bar{K}^{0}$. A simple exposition of the argument appears in section 7.1 of Nieto and Goldman [2]. If the gravitational potential $\phi_{g}$ is assumed to have absolute meaning, then the energy difference between $K^{0}$ and $\bar{K}^{0}$ due to antimatter gravitational asymmetry should be $\frac{g-\bar{g}}{g} M_{K} \phi_{g}$, which for hypothetical "antigravity" $(\bar{g}=-g)$ is $2 M_{K} \phi_{g}$. Since $K_{L}$ is a superposition of $K^{0}$ and $\bar{K}^{0}$, any $K_{L}$ in a beam should regenerate some $K_{S}$ because the relative time variation of the $K^{0}$ and $\bar{K}^{0}$ components is $\exp \left(2 i M_{k} \phi_{g} t / \hbar\right)$. Now regeneration of $K_{S}$ is observed as CP violation, and we know the size of that apparent $\mathrm{CP}$ violation. Tying all this together, we get, for some given $\phi_{g}$, a limit on $\bar{g}-g$.

Using the same time effect that we used in the cyclotron frequency argument, of $(1-\bar{g} / g)\left(G M / R c^{2}\right)$, one can infer that $|1-\bar{g} / g|$ applied to the $\bar{K}^{0}$ versus the $K^{0}$ is at most two parts in a billion [15] [13]. However, unlike the cyclotron frequency observation, this speaks mostly to the gravitational attraction on $s$ and $\bar{s}$ quarks; it is a bit of a leap to say that this proves the issue for matter versus antimatter in general.

What we have in both cases is evidence which hinges on the cosmological argument. That, in turn, hinges on: 
- CPT invariance

- the notion of a meaningful absolute gravitational potential

- the idea that the appropriate potential to use is that of the local super-cluster

While CPT invariance is rock-solid provable assuming only causality, local Lorentz invariance, and asymptotically flat space-time, it is in fact possible that the doubt about the flatness assumption (which may not be true on a cosmological scale) raises doubt about the applicability of CPT to our analyses. Kenyon [12], for example, couches his analysis in terms of a CPT-violating mass difference between the $K^{0}$ and $\bar{K}^{0}$.

Nieto and Goldman [2] question the use of an absolute gravitational potential, upon which rest the conclusions from both the cyclotron frequency and the $K^{0}-\bar{K}^{0}$ observations. For an infinite-range force, what matters are potential differences. They present a formalism that recomputes the effects using potential differences. Using this approach, Chardin and Rax [22] derives that the observed CP violation is just about the right magnitude to be alternatively explained by antigravity, i.e., $\bar{g} / g=-1$.

In the framework of the geometric picture presented by General Relativity, the simple "absolute potential" calculation is justified. But if there is antimatter gravitational asymmetry, then General Relativity is violated at least in the antimatter sector; the overall geometric picture and thus the absolute potential argument come into question. That is, in order to use the absolute potential based arguments to interpret these results as limiting possible antimatter asymmetry, one must logically start with the premise that General Relativity holds in its particulars, and thus that antimatter asymmetry cannot be present a priori.

Even assuming the validity of the use of an absolute gravitational potential, one must decide which system defines that potential. That in turn depends on what one assumes about the nature (length scale) of any possible matter-antimatterasymmetric gravity-like force. Kenyon selected the definable system giving the largest absolute potential; this is natural if one wants to avoid any long but finite length scales. But this is not unavoidable. Good's original paper [11], for example, chose the gravitational potential due to the Earth; this pushes the cyclotron frequency limit on asymmetry above the $1 \%$ level (though the kaon system limit remains below 1\%). Nieto and Goldman present models involving finite-range vector and scalar gravity fields, in which apparent violation of $\bar{g}=g$ could easily be seen in the envisioned experiment.

Ultimately, while we consider this class of observations (particularly the cyclotron frequency equality) to be indirect evidence of the equality of $g$ and $\bar{g}$ to high accuracy, we cannot consider this to be a direct observation of their ratio, nor an irrefutable indication that this equality must hold, because there are disputable steps in the analysis going from the observations to those conclusions. This interpretation is supported by the statements of Chardin and Rax, Nieto and Goldman, and also in section 5.4 of Will [4]. 


\section{Inferences From Astronomical (Anti)Neu- trino Observations}

Several observations from the neutrino sector have implications concerning antimatter gravitational asymmetry.

Gasperini [18] pointed out, in 1989, that if antineutrinos and their corresponding neutrinos have different gravitational coupling (or if the mass to gravitational force ratio differs between flavors of neutrinos), this leads to the local gravitational field contributing to the transition probability between two different flavor eigenstates. At the time, no experiments had yet observed neutrino oscillations, but the limits which were established were within an order of magnitude of what since has been observed in neutrino experiments. The analysis of such experiments combines the familiar flavor-mixing angles and mass differences with a parameter characterizing the antimatter gravitational asymmetry $\Delta \alpha$. A fit of the data sets an upper bound $\Delta \alpha<0.2$ if the gravitational potential is taken to be that of the Earth. However, if the galactic gravitational potential is used, the bound becomes $0.02 \%$.

Minakata and Nunokawa [17] study the effects of gravitational asymmetry spoiling the (now-accepted) oscillation mechanism explaining the solar neutrino "deficit." They place a limit on the possible asymmetry at the same level as does Gasperini, if they also use the galactic gravitational potential as their splitting field.

LoSecco [16] studies supernova SN1987A, and demonstrates that neutrinos and antineutrinos arrived at (within statistics) the same time (and at the same time as the photons). This implies, following the reasoning of Krauss and Tremaine [24], that the antineutrino gravitational asymmetry is less than $0.5 \%$. Again, the galactic gravitational potential plays a large role in this reasoning.

This category of observations shares the same questionable characteristic with the time-scaling experiments: a critical dependence on the use of an absolute gravitational potential. Moreover, the limits placed on gravitational asymmetry are much less stringent, and since these results are restricted to the neutrino sector, any hypothetical asymmetry due to differences in baryon and antibaryon couplings are not addressed. For these reasons, the neutrino-based observations are "dominated" by the cyclotron-frequency and kaon arguments, and need not be a concern when asking whether an effect is ruled out at the $1 \%$ level.

\section{Theoretical Obstacles to Gravitational Asymmetry}

Several potential theoretical obstacles to $\bar{g} \neq g$ come to mind:

There might be difficulty formulating a framework in which $\bar{g} \neq g$. However, in section 5 of Nieto and Goldberg [2] such frameworks are presented.

It might seem that CPT invariance forces $\bar{g}=g$. However, in the context of a non-asymptotically flat space-time, or of a possible underlying theory which may 
not exhibit locality (e.g., string theory), CPT invariance is not sacrosanct, and in fact is a principle which can and should be tested. (Indeed, many CPT tests have been done or are in progress.) The proposed experiment can be viewed as such a test. Chardin and Rax [22] express doubt that the CPT theorem can be demonstrated without modification for gravitation.

Various forms of the equivalence principle (see Will's book [4] for an extensive collection) might demand that $\bar{g}=g$. Again, absent independent proof of the WEP, that only transforms the proposed experiment into a test of the Weak Equivalence Principle in a realm previously unexplored.

The most compelling theoretical objection is in the form of a gedanken experiment first discussed by Morrison [23]. Say one starts with an $e^{+} e^{-}$pair starting at height $h_{0}$, travelling upward in a gravitational field with just enough kinetic energy to reach height $h_{1}>h_{0}$, and allows the pair to annihilate, producing two photons, at $h_{1}$. Suppose those photons are directed, by heavy and perfectly reflecting mirrors, such that they meet again at $h_{0}$, and that they photoproduce an $e^{+} e^{-}$pair at the original height $h_{0}$. Those photons will have gained energy by blueshift as they descend, so that the newly produced pair will have some computable kinetic energy, which, assuming the Equivalence Principle and symmetric gravitation, will be the same as the initial kinetic energy. If the positron (or the electron) were "deficient" from the viewpoint of gravitational attraction to the Earth, then the total energy gained by the photons as they descend would be greater than the sum of the kinetic energies needed to raise the pair to the specified height. Thus an energy non-conservation paradox would appear, forbidding the gravitational asymmetry in the first place.

Nieto and Goldman [2], in chapter 5, rethink this analysis, using the coupled equations including the Einsteinian (gravitational) field. They conclude that in pure tensor gravity, the Morrison argument goes through, and this forbids "antigravity." But they then show how gravivector and graviscalar fields can couple to lepton or baryon number without leading to a violation of energy conservation.

Chardin and Rax [22], on the other hand, carefully analyze the gedanken experiment even with only tensor gravity. They note that the microscopic version of the effect induces a vacuum instability in the presence of a gravitational field. However, they do not take this to exclude antigravity! They point out that the typical energy extractable from such a photon at the surface of the Earth would correspond to a wavelength of a light year, and that this vacuum instability is similar to the Hawking radiation effect which introduces time-asymmetry to general relativity. "Antigravity is just the tidal effect on the vacuum needed to induce the temperature suggested by the 'naive' expression of the equivalence principle...". This is certainly no proof that gravitational asymmetry should exist, but it at least refutes the idea that the Morrison gedanken experiment forbids gravitational asymmetry. 


\section{Conclusions}

We see from the above that the value of $\bar{g} / g$ has not been measured by direct observation, nor has a value other than one been irrefutably ruled out by theoretical arguments. Indirect evidence does suggest, however, that the value is one to a much greater precision than the $1 \%$ level, $10^{-4}$ or perhaps as small as $10^{-8}$. (The proposed $1 \%$ measurement can be the first step toward much more precise measurements; a measurement to one part in $10^{10}$ appears possible. But the question at hand addresses the $1 \%$ level.)

Even assuming that one accepts the premise that inequality of $\bar{g}$ and $g$ (at a level of $1 \%$ ) has been ruled out inferentially (by the time-scaling effects discussed above), no result directly precludes a $1 \%$ effect in the antihydrogen experiment. The issue then becomes whether the value of finally performing a direct observation is worth the expense and effort of the experiment. The proposers feel that it is.

To do a study of long-range violations of General Relativity in situations involving antimatter, one must make multiple observations under different circumstances; the key point would be agreement or discrepancy among the results. This is the same strategy proposed for the (billion dollar) Stage IV dark energy program [25], where multiple observations sensitive to dark energy will be compared for possible discrepancies that could, for instance, signal a problem with General Relativity on cosmological scales.

In our case, the cyclotron frequency of the antiproton is one basic observation, with length scale of the distance to the local strong galactic attractor. The direct measurement of antihydrogen deflection in the Earth's gravitational field would constitute another basic observation, on a very different length scale. Even a negative result would then become part of a true suite of experimental tests of the range of validity of General Relativity.

\section{References}

[1] T. J. Phillips, G. P. Jackson, D. M. Kaplan, et al., "Letter of Intent: Antihydrogen Gravity Experiment at Fermilab," presented to the FNAL PAC March 2008 .

[2] M. M. Nieto and T. Goldman, "The Arguments Against 'Antigravity' and the Gravitational Acceleration of Antimatter," Phys. Rep. 205, No. 5 (1991) 221.

[3] W.-M. Yao et al, "Review of Particle Physics", J. Phys. G 33 (2006), p. 205.

[4] C. Will, "Theory and Experiment in Gravitational Physics," Cambridge University Press (1993).

[5] A. Frank et al., JETP Letters 86, 225 (2007).

[6] E. Adelberger et al., "Testing the Equivalence Principle in the Field of the Earth: Particle Physics at Masses Below $1 \mu \mathrm{eV}$ ?," Phys. Rev. D 42, No. 10 (1990) 3267.

[7] V. Nesvizhevshy et al., Phys. Rev. D 67, 102002 (2003). 
[8] E. Adelberger, B. Heckel, C. Stubbs, and Y. Su, "Does Antimatter Fall with the Same Acceleration as Ordinary Matter?," Phys. Rev. Lett. 66, No. 7 (1991) 850.

[9] T. Ericson and A. Richter, "Empirical Limits to Antigravity," Europhys. Lett. 11 (4) (1990) 295-300.

[10] T. Goldman, M. M. Nieto, M. Holzscheiter, T. Darling, M. Shower, and J. Schecker, Phys. Rev. Lett. 67, No. 8 (1991) 1048.

[11] M. L. Good, Phys. Rev. 121, 311 (1961).

[12] I. R. Kenyon, "A Recalculation of the Gravitational Mass Difference Between the $K^{0}$ and $\bar{K}^{0}$ Mesons," Phys. Lett. B 237, No. 2 (1990) 274.

[13] R. Hughes and M. Holzscheiter, "Constraints on the Gravitational Properties of Antiprotons and Protons from Cyclotron-Frequency Measurements," Phys. Rev. Lett. 66, No. 7 (1991) 854.

[14] G. Gabrielse et al., Phys. Rev. Lett. 82, No. 16, 3198 (1999); 65, 1317 (1990); 63, 1360 (1989).

[15] CPLEAR Collaboration, "Tests of the Equivalence Principle with Neutral Kaons," Phys. Lett. B 452, 425 (1999).

[16] J. LoSecco, Phys. Rev. D 39, No. 4 (1989) 1013.

[17] H. Minakata and H. Nunokawa, Phys. Rev. D 51, No. 12 (1995) 6625.

[18] M. Gasperini, Phys. Rev. D 39, No. 12 (1989) 3606.

[19] B. Kayser, remarks at PAC meeting, Fermilab, March 26, 2008.

[20] T. Phillips, L. Fortney, A. Goshaw, Fermilab EOI13 (1995).

[21] M. H. Holzscheiter et al., "Are Anti-Protons Forever?," Phys. Lett. A 214, 279 (1996)

[22] G. Chardin and J.-M. Rax, "CP violation. A matter of (anti)gravity?," Phys. Lett. B 282 (1992) 256.

[23] P. Morrison, "Approximate Nature of Physical Symmetries," Am. J. Phys. 26 (1958) 358.

[24] L. Krauss and S. Tremaine, Phys. Rev. Lett. 60, No. 3 (1988) 176.

[25] A. Albrecht et al., "Report of the Dark Energy Task Force," arxiv.org/abs/astro-ph/0609591 (2006). 